REVISTA DE LA ESCUELA DE CIENCIAS DE LA EDUCACIÓN, AÑO 17, NRO. 16, VOL. 2, JULIO A DICIEMBRE DE 2021. PÁGINAS 37-46. ISSN 2362-3349 (EN LÍNEA). LAS MIRADAS DE ESTUDIANTES DEL NIVEL SECUNDARIO SOBRE LOS SABERES PARA EL TRABAJO EN EL CAPITALISMO FLEXIBLE. UN ESTUDIO EN CALETA OLIVIA, SANTA CRUZ. MAURO VICTOR GUZMÁN.

\title{
LAS MIRADAS DE ESTUDIANTES DEL NIVEL SECUNDARIO SOBRE LOS SABERES PARA EL TRABAJO EN EL CAPITALISMO FLEXIBLE: UN ESTUDIO EN CALETA OLIVIA, SANTA CRUZ
}

\author{
Mauro Victor Guzmán* \\ CONICET - Universidad Nacional de la Patagonia Austral, Argentina \\ viktorm_g@hotmail.com
}

Recibido: 27/07/2020 - Aceptado: 5/12/2020

\section{Resumen}

El artículo indaga las características de los circuitos diferenciados de formación para el trabajo en escuelas secundarias públicas de Caleta Olivia, provincia de Santa Cruz. Se tienen en cuenta los sentidos de los y las estudiantes sobre los saberes para el trabajo que se enseñan en escuelas secundarias emplazadas en distintas condiciones de pobreza urbana. Se trabaja con encuestas de carácter muestral, cuyo criterio de selección es el porcentaje de necesidades básicas insatisfechas (NBI) de los emplazamientos urbanos de las escuelas. Los resultados del artículo refieren a, por un lado, la existencia de saberes transversales no asociados al trabajo que están presentes en todas las escuelas. Por otro lado, se encontraron redes de escolarización que presentan distribuciones desiguales de saberes para el sector productivo, en correspondencia con las condiciones de pobreza en la que se emplazan las escuelas. Esta distribución de saberes para el trabajo que brindan las escuelas secundarias se encuentra en sintonía con las características del mercado sociolaboral de la región, en el cual se hacen eco los rasgos del mundo del trabajo en tiempos de capitalismo flexible.

Palabras clave: Formación para el trabajo - Saberes - Distribución desigual - Escuela secundaria Estudiantes.

\section{HIGH SCHOOL STUDENTS' VIEWS ABOUT JOB SKILLS IN FLEXIBLE CAPITALISM: A STUDY IN CALETA OLIVIA, SANTA CRUZ}

\begin{abstract}
This article investigates the characteristics of the differentiated job training circuits in high schools in Caleta Olivia, province of Santa Cruz (Argentina). The students' views about the knowledge for work that is taught in high schools located in varying degrees of urban poverty is our point of departure. Ample surveys were conducted, using the percentage of unsatisfied basic needs (UBN) of the urban schools as the selection criterion. On the one hand the results show that, the existence of transversal knowledge not associated with work is present in all schools. On the other hand, schooling networks that present unequal distributions of knowledge for the productive sector were found, showing a correspondence with the

\footnotetext{
*Profesor en Ciencias de la Educación (Universidad Nacional de la Patagonia Austral). Doctorando en Ciencias de la Educación (Universidad Nacional de La Plata).
} 
REVISTA DE LA ESCUELA DE CIENCIAS DE LA EDUCACIÓN, AÑO 17, NRO. 16, VOL. 2, JULIO A DICIEMBRE DE 2021. PÁGINAS 37-46. ISSN 2362-3349 (EN LÍNEA). LAS MIRADAS DE ESTUDIANTES DEL NIVEL SECUNDARIO SOBRE LOS SABERES PARA EL TRABAJO EN EL CAPITALISMO FLEXIBLE. UN ESTUDIO EN CALETA OLIVIA, SANTA CRUZ. MAURO VICTOR GUZMÁN.

conditions of poverty in which the schools are located. This distribution of knowledge for work provided by high schools is aligned with the characteristics of the socio-labor market in the region that reflect the features of the world of work typical of a flexible capitalism.

Keyword: Job training - Knowledge - Uneven distribution - High school - Students.

\section{Introducción}

Hasta fines de los '70 la mayoría de los debates en relación a los saberes para el trabajo giraban en torno a la división entre tareas de administración, supervisión y ejecución (Braverman, 1987; Coriat, 2011; Bowles y Gintis, 1985; Baudelot y Establet, 1975). Ello implicaba una división y jerarquización entre trabajo mental y trabajo manual, característica propia de la división social del trabajo desde el capitalismo manufacturero (Marx, 1990; Braverman, 1987) y profundizada a partir de la primera década del siglo XX en la "organización científica del trabajo" bajo el modo de producción taylor-fordista (Coriat, 2011; Harvey, 2000). En este marco, las teorías neomarxistas proponían que las relaciones sociales en la escuela se correspondían con aquellas que sucedían en el mundo de la producción (Bowles y Gintis, 1985) y, por ende, con la distribución de saberes para el trabajo desde la escuela. Así se configuraban circuitos escolares diferenciados bajo la forma de Redes de escolarización ${ }^{1}$. (Baudelot y Establet, 1975). Sin embargo, muchas de estas tesis de segmentación se han puesto en discusión a partir de investigaciones que desde fines de los 80 , y principalmente en los 90 , muestran la no correspondencia entre niveles de escolarización y puestos laborales (Braslavsky, 1985; Llomovatte, 1990; Baudelot, 1998).

En este trabajo se propone analizar la distribución de saberes para el trabajo desde lo que acontece en la formación escolar, visto desde las miradas de estudiantes de nivel secundario. En todo caso, si las relaciones sociales en la escuela se corresponden con las relaciones sociales de producción, cabe la pregunta por esas correspondencias ante las mutaciones en el mundo del trabajo en tiempos de capitalismo flexible (Sennett, 2009; Harvey, 2020). Estos interrogantes se focalizan en la región del Golfo San Jorge, específicamente en la localidad de Caleta Olivia. Aquí los ritmos y lógicas flexibles del capital transnacional producen efectos en las dinámicas socio-laborales de la localidad, a partir de las actividades extractivas de petróleo. Esta sigue siendo la base sobre la que se configura la estructura económica y las dinámicas del mercado laboral. Ello desde la generación de empleos directos en el sector petrolero, como también mediante las regalías que reciben las administraciones públicas y las posibilidades de creación de empleo en el Estado (Vacca y Shinelli, 2005; Schweitzer, 2012).

Desde este contexto regional, el artículo se propone describir y analizar las características que asumen las redes de formación para el trabajo en las escuelas secundarias públicas de Caleta Olivia, atendiendo a la distribución urbana de la pobreza². En este trabajo el foco está puesto en las percepciones de los y las estudiantes del nivel secundario acerca de la enseñanza de saberes en relación al mundo del trabajo, y sus relaciones con el emplazamiento urbano de los establecimientos educativos. Para ello, se trabaja con la georreferenciación urbana de: a) Percepciones de estudiantes de escuelas secundarias públicas de la localidad en estudio, a partir de una encuesta realizada en 2018; b) Datos de Necesidades Básicas Insatisfechas (NBI) ${ }^{3}$. que arroja el último censo del INDEC para el año 2010.

El indicador de NBI es usado para caracterizar el nivel de pobreza del radio censal en que se emplazan las escuelas de nivel secundario en Caleta Olivia. Se han agrupado los porcentajes de NBI en terciles, cuyos polos van desde "NBI Bajo" (que corresponde al tercil 1, y que contiene a los radios censales en que hay menos cantidad de hogares con pobreza), al "NBI Alto" (que corresponde al tercil 3, y que contiene a los radios censales donde hay mayor cantidad de hogares en situación de pobreza). A fin de obtener la mirada de estudiantes de los distintos terciles, se seleccionaron escuelas cuyos emplazamientos abarquen los tres agrupamientos de NBI, tal como expresa el Mapa 01.

Así, el percil NBI Bajo está compuesto por una sola institución educativa que corresponde al segundo bachillerato público más antiguo de Caleta Olivia (creado en 1977) y uno de los más demandados de la localidad en épocas de inscripciones, así como una de las instituciones que más rápidamente queda sin vacante. En esta institución se ofrecen dos orientaciones: Humanidades y Cs Sociales, y Turismo. El percil NBI Medio está compuesto por una sola institución educativa que corresponde al bachillerato público más antiguo de la localidad (creado en 1962). La escuela ofrece dos orientaciones: Humanidades y Cs Sociales, y Economía y

\footnotetext{
${ }^{1}$ Según Boudelot y Establet (1975) las redes de escolarización se conforman, por un lado, por la Red Primaria Profesional a donde concurren los hijos de la clase obrera y donde se les inculcan saberes basados en las actividades de repetición y en la sumisión, y cuya preparación se dirige exclusivamente al trabajo manual o fabril. Y, por otro lado, la Red Secundaria Superior, dirigida a la formación de los hijos de la burguesía y cuya formación gira en torno a saberes asociados a la abstracción y a la promoción de los futuros intérpretes activos de la ideología burguesa.

${ }^{2}$ Los datos que aquí se presentan corresponden al trabajo de investigación realizado en el marco general del PDTS "Centro de Intercambio y Reservorio de Información Social y Educativo (CIRISE)" (http://www.uaco.unpa.edu.ar/cirise/index.php) alojado en el Area Sociopedagógica de la UNPA-UACO. Asimismo, es parte de la investigación de tesis doctoral en curso, en el marco de beca CONICET, "Formación para el trabajo en el espacio urbano fragmentado: Un estudio en escuelas secundarias del Golfo San Jorge" (dirigida por Dra. Silvia Grinberg y co-dirigida por Dr. Eduardo Langer).

${ }^{3}$ Dicho indicador es un método directo para identificar carencias críticas en una población y caracterizar la pobreza, que usualmente utiliza indicadores directamente relacionados con cuatro áreas de necesidades básicas de las personas (vivienda, servicios sanitarios, educación básica e ingreso mínimo), información que se obtiene de los censos de población y vivienda (Feres y Mancero, 2001)
} 
REVISTA DE LA ESCUELA DE CIENCIAS DE LA EDUCACIÓN, AÑO 17, NRO. 16, VOL. 2, JULIO A DICIEMBRE DE 2021. PÁGINAS 37-46. ISSN 2362-3349 (EN LÍNEA). LAS MIRADAS DE ESTUDIANTES DEL NIVEL SECUNDARIO SOBRE LOS SABERES PARA EL TRABAJO EN EL CAPITALISMO FLEXIBLE: UN ESTUDIO EN CALETA OLIVIA, SANTA CRUZ. MAURO VICTOR GUZMÁN.

Administración. Por último, el percil NBI Alto está compuesto por dos instituciones educativas que fueron creadas en el 2013, últimos bachilleratos públicos que se crearon en la localidad. Las dos escuelas ofrecen sólo una orientación: una de ellas ofrece la orientación de Artes Visuales y la otra tiene la orientación de Ciencias Sociales.

Mapa 01. Ubicación de las escuelas secundarias seleccionadas, según tercil de NBI del radio censal. Caleta Olivia, 2018

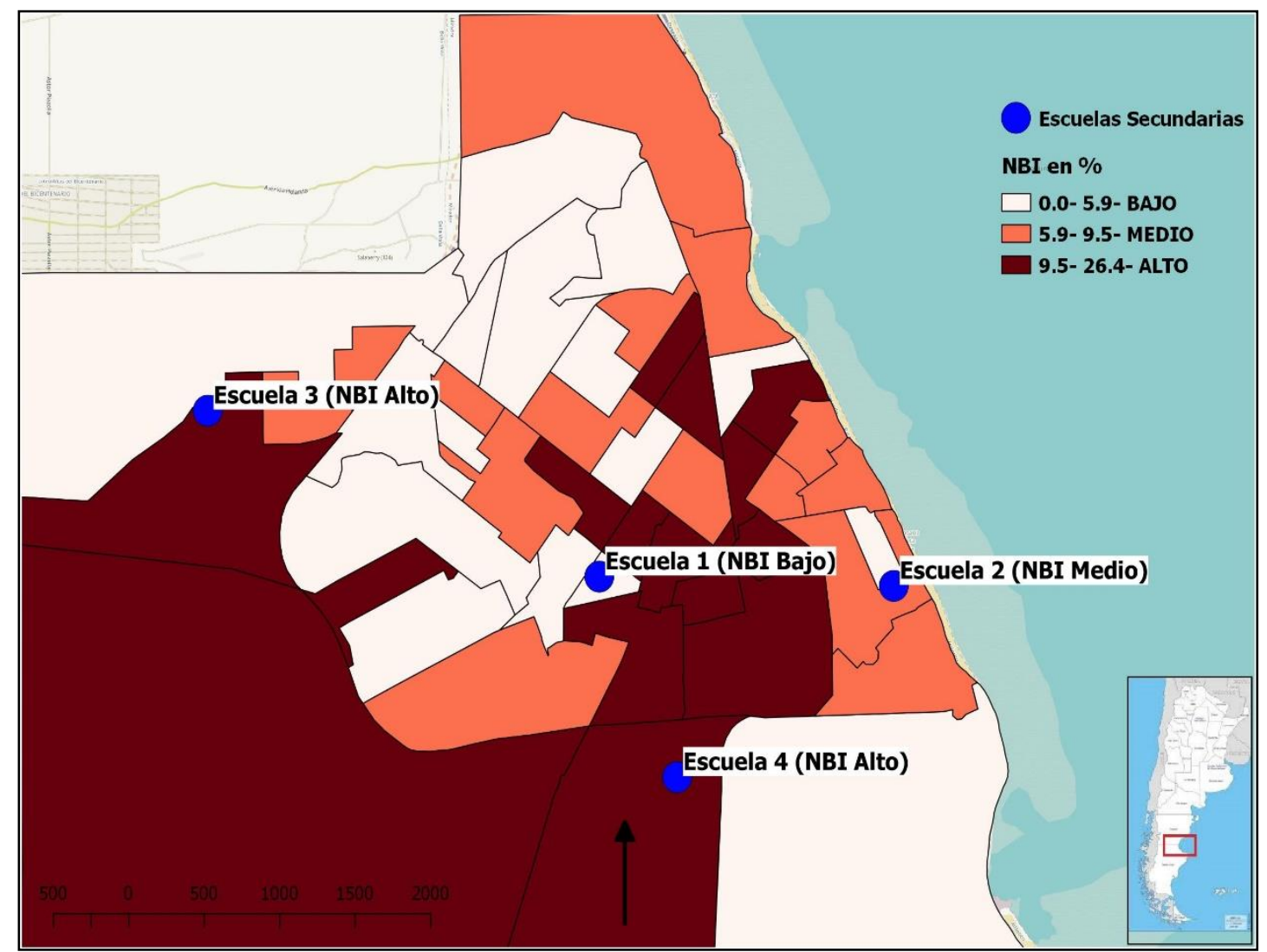

Fuente: Elaboración propia sobre la base de datos de PDTS "Centro de Intercambio y Reservorio de Información Social y Educativo (CIRISE)", alojado en el Área Sociopedagógica de la UNPA-UACO

En estas escuelas se realizaron encuestas a estudiantes que en el 2018 se encontraban cursando el primer año del Ciclo Básico (1er año del nivel secundario), el primer año del Ciclo Orientado (3er año del nivel secundario) y quienes se encontraban en el último año del Ciclo Orientado (5to año en bachilleratos). Este criterio buscó abarcar la mirada de los y las estudiantes considerando los momentos claves relacionados con el inicio del nivel secundario en general, el inicio del ciclo orientado y la perspectiva de quienes han transitado por todo el nivel y están a punto de finalizar la secundaria. El instrumento de encuesta estuvo compuesto por distintas dimensiones de análisis que refirieron a las vivencias de los y las estudiantes sobre su escolaridad y perspectivas a futuro relacionadas con ello. Para este trabajo, se abordan sus respuestas sobre las valoraciones de cómo la escuela los prepara para ciertos saberes, así como por la indagación de saberes vinculados al trabajo que consideran que la escuela les está enseñando. Estas miradas son leídas en estrecha relación con las características socio-urbanas de emplazamiento escolar y también del contexto socio-laboral de Caleta Olivia.

De esta forma, el trabajo se estructura en tres partes. En primer lugar, se desarrollan abordajes teóricos y conceptuales sobre las mutaciones del trabajo en el capitalismo flexible, los efectos en la desigualdad social y urbana y, principalmente, las implicancias en los saberes para el trabajo. Luego, se menciona cómo estos procesos se hacen eco en las dinámicas socio-laborales de Caleta Olivia. En un segundo momento, se abordan las miradas de los y las estudiantes de escuelas secundarias de la localidad en estudio. Allí se analiza cómo ellos y ellas consideran los saberes que se enseñan en la escuela y sus utilidades para el mundo del trabajo, comparando estos sentidos según el emplazamiento urbano de la escuela. Por último, se presentan reflexiones en relación a las formas en que se constituyen las redes de formación para el trabajo desde las escuelas secundarias en Caleta Olivia, cómo estas se relacionan con las dinámicas de fragmentación urbana y las correspondencias con el mercado socio-laboral de la región en tiempos de capitalismo flexible. 


\section{Las dinámicas sociolaborales de la cuenca del Golfo San Jorge y la formación para el trabajo}

Desde fines del siglo pasado, las teorizaciones y conceptualizaciones sobre el capitalismo flexible (Harvey, 2000; Sennett, 2000), la crisis de la sociedad salarial (Castel, 1997), el devenir de una gubernamentalidad neoliberal (Foucault, 2007), entre otras, han instalado una serie de debates en torno a las mutaciones en el mundo del trabajo y los saberes para el trabajo. En Latinoamérica, diversos estudios han mostrado que el proceso de precarización del trabajo tiene un impacto con características particulares. Ello a partir de considerar que para esta región han coexistido formas ancestrales de precariedad laboral con el Estado de Bienestar, cuya crisis ha visto combinar estas condiciones pre-existentes con nuevas formas de precariedad (De la Garza, 2000; Olivera, Alfaro y Estrada, 2012; Jacinto, 2013; De Ibarrola, 2016). Miranda y Arancibia (2017) manifiestan que uno de los factores específicos de la región latinoamericana para dar cuenta de los procesos de desigualdad social, educativa y de las juventudes está asociado a la idea de espacialidad urbana y segregación territorial. Distintos estudios vienen mostrando cómo los procesos de urbanización y las prácticas y usos del espacio urbano producen en éste una distribución de la pobreza ya no desde una lógica centro-periferia, uniforme y/o segmentada. Mas bien, la configuración del espacio o modelo de ciudad en la región viene siendo conceptualizada en términos de fragmentación urbana (Prevot-Shapira, 2001; De Marinis, 2002; Janoshka, 2004; Veiga, 2009; Carman, Vieira da Cunha y Segura, 2013; Saraví, 2015).

Considerando que estos procesos refieren a dinámicas globales y regionales, y a fin de dar cuenta de qué manera estos escenarios globales están presentes en escenarios locales, cabe una breve caracterización del contexto socio-laboral en la región en que se ubica la localidad en estudio. Caleta Olivia es una de las localidades que forman parte de la Cuenca del Golfo San Jorge. Dicho espacio regional se crea y desarrolla a partir de la extracción de petróleo como matriz productiva, la cual ha generado un proceso de crecimiento desde principios de siglo XX en las localidades que la conforman (Salvia, 1997; Acconcia y Muñoz, 2008). Aquí la noción de enclave (Salvia, 1997) cobra relevancia en la medida en que no sólo refiere al desarrollo económico, sino también al conjunto de relaciones sociales asalariadas que se configuraron en las localidades de la región. De allí que la privatización de Yacimientos Petrolíferos Fiscales (YPF) y la reconfiguración del proceso productivo bajo las lógicas flexibles del capital a fines del siglo XX, produjeron efectos desestructurantes en el mercado de trabajo y los trabajadores (Salvia, 1997; Ruiz y Muñoz, 2008). Estos efectos se han expresado, y aún están presentes, en las sucesivas huelgas y prácticas de lucha en general por parte de trabajadores del propio sector petrolero y de la administración pública, como también de movimientos de desocupados, las cuales han llegado a colocar a Caleta Olivia como "capital nacional del piquete" (Schweitzer, 2012; Marquez, 2017).

En los últimos años la estructura económica y el mercado laboral en el Golfo San Jorge siguen configurándose en torno a la actividad petrolera, ya sea de forma directa como también mediante las regalías que reciben las administraciones públicas y las posibilidades de creación de empleo en el Estado (Vacca y Shinelli, 2005; Schweitzer, 2012). Siguiendo a Vacca y Schinelli (2005), dado que las principales empresas se concentran en propietarios extranjeros, existe una gran dependencia de factores económicos internacionales, generando una necesidad de adaptarse a la coyuntura internacional y a las decisiones de sus accionistas. Al respecto, señala Marquez (2017) que esto es constitutivo de las actuales tensiones entre, por un lado, la búsqueda de aumento de rentabilidad mediante reducción de trabajo humano excedente por parte de las empresas y, por otro, las disputas por evitar los despidos por parte de los trabajadores. Esta matriz económica y laboral de la región va conformando sociedades que se componen por:

(...) sectores vinculados de alguna manera a la actividad extractiva, con ingresos más altos, otros empleados en el sector público, con ingresos muchos menores y sufriendo el impacto de subas de precios de vivienda y bienes de consumo básico y, finalmente, otros que no encuentran inserción y protagonizan lógicas de protestas por trabajo o son víctimas del clientelismo a cambio de subsidios 0 bolsas de comida (Schweitzer, 2012, p. 47-48).

Con lo cual, en Caleta Olivia, y en la región del Golfo San Jorge en general, las dinámicas del capitalismo flexible se hacen eco en el mundo del trabajo de la región. Ello a partir de una economía que aún se configura alrededor de la actividad petrolera, sujeta a la coyuntura internacional y las lógicas flexibles del capital global (Vacca y Shinelli, 2005; Schweitzer, 2012; Marquez, 2017). Por ello, pensar la formación para el trabajo desde la escuela, y específicamente en las escuelas secundarias de Caleta Olivia, implica dar cuenta de cómo se configuran los saberes para el trabajo y las discusiones sobre los procesos de formación en tiempos de capitalismo flexible.

Una de las particularidades de dicho modo de producción refiere a cómo se organiza la división (o más bien la fusión) entre trabajo manual y trabajo mental, como parte de la organización de las tareas. Dicha división de la fuerza de trabajo entre actividades manuales y actividades mentales tiende a difuminarse a partir de la incorporación de la microelectrónica a los procesos productivos: las tareas más simples y repetitivas tienden a ser reemplazadas por el complejo tecnológico capaz de realizarlas (Coriat, 2011). Esto ha llevado a que las tareas de las que se ocupa el trabajador requieran cada vez más de saberes ligados a la planificación, control, ajuste y mantenimiento. Según Lazzarato y Negri (2001), en este proceso de inmaterialización del modo de producción capitalista la actividad abstracta que remite a la subjetividad del trabajador tenderá a ser dominante. Ello ha producido un desplazamiento de la imagen del trabajador fabril hacia otras construcciones vinculadas al trabajo intelectual. En este sentido, Grinberg (2003), analizando los significados del trabajo desde los campos de producción curricular en la escuela, menciona el pasaje del "obrero" al "operario". Por otro lado, visto desde el 
REVISTA DE LA ESCUELA DE CIENCIAS DE LA EDUCACIÓN, AÑO 17, NRO. 16, VOL. 2, JULIO A DICIEMBRE DE 2021. PÁGINAS 37-46. ISSN 2362-3349 (EN LÍNEA). LAS MIRADAS DE ESTUDIANTES DEL NIVEL SECUNDARIO SOBRE LOS SABERES PARA EL TRABAJO EN EL CAPITALISMO FLEXIBLE: UN ESTUDIO EN CALETA OLIVIA, SANTA CRUZ. MAURO VICTOR GUZMÁN.

lenguaje de las organizaciones empresariales, las mutaciones de la noción de "obrero/operario" al de "operadores" (Figari, 2011).

Con estos sentidos, algunos estudios en la región del Golfo San Jorge han mostrado cómo la incorporación de tecnologías cada vez más avanzada en la actividad petrolera desde fines de los '90, vienen produciendo una reconfiguración del trabajo y las disposiciones que se demandan de los trabajadores, las cuales se encuentran sujetas a la incorporación del paradigma informacional de creación de valor (Aranciaga, 2004). Es decir, se ha generado un desplazamiento de la imagen del obrero petrolero definido exclusivamente por actividades manuales y repetitivas (Acconcia y Alvarez, 2008), hacia otras identificaciones en términos de polivalencia o "tareas múltiples" que implican mayores actividades de abstracción (Aranciaga, 2004). El quiebre entre estos saberes para el trabajo con aquellos que caracterizaban al obrero petrolero de YPF, asociados a la especialización en un oficio vinculado al hacer manual y corporal (Acconcia y Alvarez, 2008), expresaron el modo en que las lógicas del capitalismo flexible se hicieron eco en las "nuevas necesidades" de saberes para el trabajo:

(...) los ex trabajadores de YPF entendían, agónicamente, que sus saberes del trabajo sólo eran aplicables al mundo laboral del que provenían (...) La gestión sostenida en la intensificación del trabajo ligada a la polivalencia y la multifuncionalidad, trajo aparejada la necesidad de capacitación permanente y la formación en el puesto de trabajo (Ruiz y Muñoz, 2008, p.111-113).

Esta demanda de un trabajador polivalente y multifuncional expresa, entre otras cosas, el desplazamiento de la concepción de saberes para el trabajo en términos de cualificación, hacia la noción de saberes asociados a competencias (Tanguy, 1986; Spinosa, 2006; Jacinto y Millenaar, 2012; Brunet y Zavaro, 2014). La noción de cualificación estaba asociada a los saberes que referían a los trabajadores centrados en tareas específicas y donde la duración acotada del aprendizaje aparecía como uno de los elementos constitutivos de estos procesos (Harvey, 2000; Brunet y Zavaro, 2014). En cambio, a través de la noción de competencias se entiende como:

(...) un modo de funcionamiento integrado de la persona, en el que se articulan recursos tales como: conocimientos, habilidades, actitudes, aptitudes, valores, así como los procesos motivacionales, emocionales, afectivos y volitivos, en el desempeño de la profesión/ocupación, y que provee a la persona, de la posibilidad de tomar decisiones inteligentes en situaciones que son suficientemente nuevas (Brunet y Zavaro, 2014, p. 12).

Es decir, el concepto de competencias encuentra su especificidad en la cuestión del Saber Ser y cómo se concibe el Saber Hacer (Spinosa, 2006; Grinberg, 2008; Jacinto y Millenaar, 2012; Brunet y Zavaro, 2014). Noguera Ramírez y Marín Diaz (2012) señalan que hoy el aprendizaje con énfasis en la gubernamentalidad" neoliberal se centra en las competencias de aprender a conocer, aprender a hacer, aprender a vivir y aprender a ser. Estos saberes y aprendizajes se constituyen en disposiciones y habilidades que los sujetos son llamados a poseer, y las instituciones educativas a enseñar, a fin de que cada uno pueda gerenciar las propias posibilidades, de invertir en cierto capital humano, de ser el gestor de la propia biografía (Grinberg, 2008; Castro Gómez, 2010; Jacinto y Millenaar, 2012). Según Foucault (2007), esta interpelación a la dimensión subjetiva del trabajador, no considerada por el liberalismo y los economistas clásicos en general, nos habla de una de las características propias de la gubernamentalidad en nuestros tiempos. En el neoliberalismo los procesos de subjetivación se dirigen a formar un sujeto bajo la figura del "empresario de sí mismo" (Foucault, 2007; Castro Gómez, 2010; Noguera Ramirez y Marin Diaz, 2012). Este sujeto es llamado a gestionar su propio bienestar y los medios para conseguirlos. Entre ellos se encuentra el de invertir en su propia formación, tal como lo proponen las versiones clásicas y actuales de la teoría del capital humano, y que se conceptualizan bajo la noción de competencias que se ha mencionado.

Ambas figuras, la del trabajador polivalente y la del empresario de sí mismo, pueden ser complementarias para analizar las características que asumen los procesos de formación desde la escuela. Asimismo, nos proponen el desafío de pensar los modos en que se constituyen las correspondencias entre los saberes que circulan en las escuelas, y las dinámicas de un mundo del trabajo inestable, flexible y que nos interpela a adquirir competencias a fin de cambiar y adaptarnos constantemente. A continuación, daremos cuenta de estos interrogantes focalizados en la mirada de estudiantes que cursan el nivel secundario en la localidad petrolera de Caleta Olivia.

\section{La formación para el trabajo en la escuela desde la mirada de estudiantes}

En el marco de los debates presentados, las miradas que tienen los y las estudiantes sobre la formación en saberes que desarrolla la escuela tienen un particular interés. Nos permiten dar cuenta de los modos en que los procesos de subjetivación van construyendo sentidos con respecto al lugar que la escuela tiene en y durante ese proceso de formación para el trabajo, y las utilidades de los saberes que allí circulan.

\footnotetext{
${ }^{4}$ La gubernamentalidad remite al modo de gobierno de la población, entendiendo a este como un arte, como una manera de
} dirigir y guiar la conducta de los sujetos (Foucault, 2007). 
REVISTA DE LA ESCUELA DE CIENCIAS DE LA EDUCACIÓN, AÑO 17, NRO. 16, VOL. 2, JULIO A DICIEMBRE DE 2021. PÁGINAS 37-46. ISSN 2362-3349 (EN LÍNEA). LAS MIRADAS DE ESTUDIANTES DEL NIVEL SECUNDARIO SOBRE LOS SABERES PARA EL TRABAJO EN EL CAPITALISMO FLEXIBLE: UN ESTUDIO EN CALETA OLIVIA, SANTA CRUZ. MAURO VICTOR GUZMÁN.

En lo que sigue, nos centramos en indagar las miradas de los y las estudiantes con respecto a la formación en saberes en general y aquellos relacionados al mundo del trabajo en particular, considerando las condiciones de emplazamiento de los establecimientos educativos. Así, la Tabla № 1 expresa los sentidos que los y las estudiantes han manifestado sobre la valoración de cómo la escuela los y las prepara en ciertos saberes:

Tabla $N^{\circ}$ 1. Grados de valoración de la preparación de la escuela según los y las estudiantes y por NBI de radio escolar de Caleta Olivia, 2018. En \% ( $=212)$.

\begin{tabular}{|c|c|c|c|c|}
\hline \multirow{2}{*}{ SABERES } & \multirow{2}{*}{ VALORACION } & \multicolumn{3}{|c|}{ NBI } \\
\hline & & Bajo & Medio & Alto \\
\hline \multirow{3}{*}{ TRABAJO EN EQUIPO } & Bien o Muy Bien & 80,8 & 68,3 & 79,1 \\
\hline & Regular o Mal & 19,2 & 31,7 & 20,9 \\
\hline & Total & 100 & 100 & 100 \\
\hline \multirow{3}{*}{ EXPONER ANTE UN GRUPO } & Bien o Muy Bien & 81,6 & 63,4 & 76,9 \\
\hline & Regular o Mal & 18,4 & 36,6 & 23,1 \\
\hline & Total & 100 & 100 & 100 \\
\hline \multirow{3}{*}{ USAR TECNICAS DE ESTUDIO } & Bien o Muy Bien & 77,9 & 76,7 & 72,6 \\
\hline & Regular o Mal & 22,1 & 23,3 & 27,4 \\
\hline & Total & 100 & 100 & 100 \\
\hline \multirow{3}{*}{ BUSCAR INFORMACION } & Bien o Muy Bien & 91 & 90 & 84,1 \\
\hline & Regular o Mal & 9 & 10 & 15,9 \\
\hline & Total & 100 & 100 & 100 \\
\hline \multirow{3}{*}{ USAR LAS TECNOLOGIAS } & Bien o Muy Bien & 75,7 & 46,3 & 62,5 \\
\hline & Regular o Mal & 24,3 & 53,7 & 37,5 \\
\hline & Total & 100 & 100 & 100 \\
\hline \multirow{3}{*}{ COORDINAR UN GRUPO DE TRABAJO } & Bien o Muy Bien & 64,9 & 66,7 & 69 \\
\hline & Regular o Mal & 35,1 & 33,3 & 31 \\
\hline & Total & 100 & 100 & 100 \\
\hline \multirow{3}{*}{ VALORAR LA CULTURA DEL TRABAJO } & Bien o Muy Bien & 76 & 69 & 76,2 \\
\hline & Regular o Mal & 24 & 31 & 23,8 \\
\hline & Total & 100 & 100 & 100 \\
\hline \multirow{3}{*}{ VALORAR EL ESFUERZO QUE HAGO PARA SUPERARME } & Bien o Muy Bien & 80,5 & 83,3 & 81,5 \\
\hline & Regular o Mal & 19,5 & 16,7 & 18,5 \\
\hline & Total & 100 & 100 & 100 \\
\hline
\end{tabular}

Fuente: Elaboración propia sobre la base de datos de PDTS "Centro de Intercambio y Reservorio de Información Social y Educativo (CIRISE)", alojado en el Área Sociopedagógica de la UNPA-UACO

La Tabla № 1 expresa que para una considerable cantidad de estudiantes la escuela los prepara bien o muy bien, y esto ocurre para todas las instituciones en los distintos terciles de NBI de emplazamiento escolar. Aun así, hay encuentros y desencuentros de valoración de saberes según condiciones de pobreza en que se ubica la escuela, y aquí es donde se pondrá el foco a continuación.

Con respecto a los encuentros y semejanzas, el primer dato a destacar es que en los tres NBI, el saber que mayormente es valorado por los y las estudiantes es el de Buscar Información. Como se ha mencionado, una de las competencias centrales en nuestros tiempos refiere no tanto a la adquisición de conocimientos sino a la capacidad de llegar a ellos (Noguera Ramírez y Marín Díaz, 2012). Al respecto, Grinberg (2013) señala que, en el marco del paradigma de la sociedad de la información, y ante el imperativo del aprender a aprender, el deber ser de la acción educativa se constituye en la formación de un Yo buscador:

(...) es posible entender cómo docentes y/o estudiantes hemos devenido sujetos llamados a dejar de enseñar y aprender información, en tanto los contenidos conceptuales se han visto devaluados; en aras de volvernos sujetos activos de nuestro propio aprendizaje, buscar información y participar en esa búsqueda constante se ha vuelto el eje de la tarea educativa (Grinberg, 2013, p. 93). 
REVISTA DE LA ESCUELA DE CIENCIAS DE LA EDUCACIÓN, AÑO 17, NRO. 16, VOL. 2, JULIO A DICIEMBRE DE 2021. PÁGINAS 37-46. ISSN 2362-3349 (EN LÍNEA). LAS MIRADAS DE ESTUDIANTES DEL NIVEL SECUNDARIO SOBRE LOS SABERES PARA EL TRABAJO EN EL CAPITALISMO FLEXIBLE: UN ESTUDIO EN CALETA OLIVIA, SANTA CRUZ. MAURO VICTOR GUZMÁN.

El saber buscar información se produce transversamente en todas las escuelas, más allá de las condiciones de emplazamiento escolar. En esa línea, también es necesario resaltar la importancia que otorgan al saber Valorar el esfuerzo que hago para superarme en las escuelas de los tres NBI. Éste corresponde al segundo saber con mayor valoración en las escuelas de NBI Medio y NBI Alto y el tercero en NBI Bajo. Buscar información, así como valorar el esfuerzo son saberes transversales, más allá de todo emplazamiento, que se integran como complementos claves en la formación de ese Yo buscador. En estos tiempos no sólo hay que saber buscar, sino también tener la actitud y voluntad para hacerlo y, principalmente, sobreponerse a las frustraciones de no encontrar (Grinberg, 2008; Noguera Ramirez y Marin Diaz, 2012; Jacinto y Millenaar, 2012). Saber ser un buscador y valorar el esfuerzo para llevarlo a cabo son los saberes que mayormente circulan en todas las escuelas secundarias de nuestra muestra, más allá de los niveles de pobreza del emplazamiento escolar. Estos forman parte de los atributos generales del trabajador que se pretende formar en tiempos de capitalismo flexible, cuyas lógicas, como se ha mencionado, se hacen eco en la economía y mercado laboral de Caleta Olivia.

Con respecto a los desencuentros o las diferencias, el saber que tiene menor valoración en su enseñanza como también mayor disparidad entre los tres NBI, aunque no de manera lineal, es el de Usar las Tecnologías. Este es el saber menor valorado como un saber que se enseña por los estudiantes de NBI Alto (62,5\%); es el único saber en que la mayoría considera que los preparan "Mal o Regular" en NBI Medio (53,7\%); y es el segundo menos valorado por los estudiantes de NBI Bajo $(75,7 \%)$. Distintos estudios han mostrado cómo los procesos de la puesta en acto (Ball, et al., 2012) de políticas de inclusión digital vienen produciendo efectos en las instituciones educativas de nivel secundario. Estos han sido caracterizados ya sea desde la puesta en valor de la instrumentalización (Benítez Larghi, Lemus y Welschinger Lascano, 2014), como también movimientos que van de la ilusión al desencanto en la cotidianeidad escolar en contextos de pobreza urbana (Armella y Langer, 2020). Claramente, el uso de las tecnologías se vuelve cada vez más un saber clave, con implicancias en relación a un mundo del trabajo global y regional que requiere la incorporación del paradigma informacional de creación de valor (Aranciaga, 2004).

Ahora bien, estos saberes que se vienen describiendo los y las estudiantes no los piensan en relación al mundo del trabajo. Es decir, son miradas de los y las estudiantes que han valorado la enseñanza de la escuela sobre esos saberes en sí mismos. La Tabla № 2, en cambio, expresa las miradas de los y las estudiantes en relación a saberes que la escuela les enseña específicamente en relación al mundo del trabajo.

Tabla $\mathbf{N}^{\circ}$ 02. Saberes para el trabajo que enseña la escuela según los y las estudiantes, por NBI de radio escolar de Caleta Olivia. En \% ( $\mathrm{N}=\mathbf{2 1 2})$.

\begin{tabular}{|c|c|c|c|}
\hline SABERES PARA EL MUNDO DEL TRABAJO & \multicolumn{2}{|c|}{ NBI } & Alto \\
\cline { 2 - 4 } & Bajo & Medio & 23,5 \\
\hline Disciplina & 65,1 & 81,8 & 30,6 \\
\hline Saberes básicos y generales & 37,3 & 22,7 & 3,5 \\
\hline Estudiar/Información & 15,7 & 18,2 & 7,1 \\
\hline Trabajar en grupo y compañerismo & 15,7 & 25 & 0 \\
\hline Uso de las TICs & 3,6 & 2,3 & 0 \\
\hline Otros idiomas (Ingles) & 3,6 & 2,3 & 1,2 \\
\hline Esforzarse/Sacrificio & 3,6 & 4,5 & 14,1 \\
\hline Helacionado a la orientación y el título & 14,5 & 9,1 & 1,2 \\
\hline Saberes por mi cuenta & 3,6 & 0 & 8,2 \\
\hline Buena persona/Buen ciudadano & 2,4 & 11,4 & 5,9 \\
\hline Competencias comunicativas & 6 & 9,1 & 4,7 \\
\hline Pensar/Solucionar problemas & 12 & 11,4 & 2,4 \\
\hline No lo sé & 0 & 4,5 & 3,5 \\
\hline Todo me sirve & 12 & 0 & 5,9 \\
\hline En nada & 3,6 & 4,5 & 2,4 \\
\hline No responde & 1,2 & 6,8 & 42,3 \\
\hline TOTALES & 15,6 & 13,6 & $\mathbf{1 0 0} \%$ \\
\hline
\end{tabular}

Fuente: Elaboración propia sobre la base de datos de PDTS "Centro de Intercambio y Reservorio de Información Social y Educativo (CIRISE)", alojado en el Área Sociopedagógica de la UNPA-UACO

El primer dato a destacar de la Tabla № 2 es la diferencia que presenta la valoración que los y las estudiantes hacen de los saberes en sí y la consideración de estos cuando se los coloca en relación al mundo del trabajo. En los saberes que aparecen en ambas tablas y en las miradas de los y las estudiantes de las escuelas ubicadas en todos los NBI, la consideración de los saberes en sí disminuye cuando se los piensan como saberes para el trabajo. Aquí cobra fuerza la incertidumbre que caracteriza al mercado del trabajo: el 
problema del déficit de lugares ocupables, la inempleabilidad de los calificados, la relevancia de las competencias por sobre la cualificación y que, como se ha mencionado, forman parte del escenario del mercado socio-laboral de Caleta Olivia. Es decir, los y las estudiantes se preguntan "¿cuáles de los saberes que la escuela me está enseñando hoy pueden ser útiles para el trabajo el día de mañana?". Esta pregunta pareciera no tener una respuesta inmediata y mucho menos definitiva en un mundo que exige cambiar, actualizar y aprender a aprender nuevos saberes constantemente.

Aun así, los y las estudiantes otorgan valor a ciertos saberes para el trabajo que desde la escuela se les está enseñando, y éstos sí mayoritariamente varían según las condiciones de pobreza del emplazamiento escolar. El primero de ellos a resaltar es el saber asociado a la Disciplina, es decir llegar a horario, cumplir en tiempo y forma, responsabilidad, etc. En la Tabla $N^{\circ} 02$ se observa que es el saber con mayor porcentaje en las miradas de los y las estudiantes de las escuelas en NBI Bajo y NBI Medio. Es decir, visto desde las perspectivas de ellos y ellas pareciera que, en estos sectores, la formación para el trabajo desde la escuela sigue ocurriendo principalmente desde la lógica disciplinaria en el gobierno de la fuerza de trabajo (Foucault, 1989; Bowles y Gintis, 1985). Sin embargo, como mencionamos, esto no ocurre de igual manera en todas las escuelas. En aquellas ubicadas en NBI Alto el porcentaje es marcadamente menor. Allí pareciera que la disciplina se flexibiliza, siendo las características del aprendizaje con énfasis en la gubernamentalidad neoliberal (Noguera Ramirez y Marin Diaz, 2012) la lógica que prevalece en la formación para el trabajo de los sectores emplazados en zonas con mayor pobreza. Al respecto, Grinberg (2009) señala que esto es un rasgo característico de la cotidianeidad escolar en contextos de marginalidad urbana, con sus respectivas implicancias en relación al trabajo: "Así, al igual que quien ha quedado desocupado, el tiempo de la vida escolar transita aletargadamente, ya no hay dónde llegar, dónde cumplir horario, no hay un tiempo que aprovechar." (p. 176).

En esta misma línea, la consideración sobre la preparación para el Trabajo en grupo y el Estudiar asociado a la capacitación constante y, por tanto, a las competencias, también expresan diferencias según NBI de emplazamiento escolar. Sin duda, como mencionamos, estos saberes forman parte neurálgica de los procesos productivos en el capitalismo flexible, y están presentes en las demandas de los perfiles laborales en los distintos sectores de la economía regional (Rojo y Rotondo, 2009; Romero y Galaretto, 2013). Nuevamente, los porcentajes son menores en estudiantes de las escuelas ubicadas en NBI Alto. Si bien en lo que respecta al Uso de las TIC hay mayor cercanía entre las miradas de estudiantes en los tres NBI, dado que el porcentaje es bastante bajo en todas las escuelas, en los y las estudiantes de NBI Alto directamente no aparece como un saber asociado al trabajo. En el apartado anterior se mencionó que el desarrollo de la microelectrónica y los avances tecnológicos han generado mutaciones en las características del trabajo y la organización del proceso productivo (Coriat, 2011; Harvey, 2020). En el escenario regional resulta central el manejo de estas herramientas para, al menos, aspirar a ocupar un lugar en los sectores más demandados del mercado de empleo, ya sea en la actividad petrolera, el empleo público o el desarrollo de PyMES (Aranciaga, 2004; Prado y Robledo, 2010; Marquez, 2017). En correspondencia, no sólo que las escuelas de Caleta Olivia se proponen enseñar estos saberes, sino que la mayoría de los y las estudiantes expresan que se los enseñan Bien o Muy Bien (Ver Tabla 01). Pero pareciera que, de acuerdo con las miradas de estos mismos estudiantes, su enseñanza en relación al trabajo sucede diferencialmente según el emplazamiento o directamente no sucede en las escuelas emplazadas en las zonas más pobres.

Con lo cual, las redes de escolarización en las escuelas secundarias de Caleta Olivia se tornan ciertamente explicitas cuando indagamos los sentidos de los y las estudiantes sobre los saberes que la escuela les enseña para el mundo del trabajo. La conformación de estos circuitos escolares en que se distribuyen diferencialmente los saberes para el trabajo asociados al trabajo en grupo, el estudiar, el uso de las TIC e incluso la disciplina dan cuenta de ello. Estas diferenciaciones y desigualdades ubican a los y las estudiantes de las escuelas emplazadas en NBI Alto como potenciales trabajadores de las zonas más periféricas del mercado de trabajo (Harvey, 2020), o directamente como los futuros supernumerarios (Castel, 1997). Esta hipótesis se refuerza si tenemos en cuenta que solamente en la consideración de los y las estudiantes de las escuelas en NBI Alto la respuesta No responde es la que tiene más peso. Si como se mencionó, entre la consideración de la preparación de los saberes en sí y la enseñanza de esos saberes para el trabajo existe una disminución en todas las escuelas de la muestra, para los y las estudiantes de escuelas en NBI Alto ese hiato se profundiza aún más. Estos sentidos estudiantiles sobre la formación escolar, los saberes y sus utilidades para el trabajo se debe leer en relación al contexto socio-laboral del Golfo San Jorge, en que las características del mercado de trabajo se asientan sobre el carácter inestable, incierto y fluctuante propio de las lógicas del empleo y el desempleo en tiempos de capitalismo flexible.

\section{Reflexiones finales}

En este trabajo se buscó describir las características de las redes de formación para el trabajo desde las escuelas secundarias de Caleta Olivia, a partir de las miradas de los y las estudiantes. Las lecturas que se han realizado sobre estas perspectivas se enmarcan en los debates sobre las mutaciones del trabajo en el capitalismo flexible, los saberes asociados a la noción de competencias y los procesos de fragmentación urbana. Ello en un contexto regional en que las características socio-laborales presentan signos asociados a estos procesos globales. En este sentido, el interrogante sobre cómo se constituyen en Caleta Olivia los circuitos diferenciados de formación para el trabajo desde las escuelas secundarias, es abordado en relación a procesos de fragmentación urbana (Veiga, 2009; Saraví, 2015) que podrían estar formando parte del paisaje urbano de la 
REVISTA DE LA ESCUELA DE CIENCIAS DE LA EDUCACIÓN, AÑO 17, NRO. 16, VOL. 2, JULIO A DICIEMBRE DE 2021. PÁGINAS 37-46. ISSN 2362-3349 (EN LÍNEA). LAS MIRADAS DE ESTUDIANTES DEL NIVEL SECUNDARIO SOBRE LOS SABERES PARA EL TRABAJO EN EL CAPITALISMO FLEXIBLE. UN ESTUDIO EN CALETA OLIVIA, SANTA CRUZ. MAURO VICTOR GUZMÁN.

localidad (Pérez, 2013). Ello implicó analizar los sentidos de los y las estudiantes prestando atención a las condiciones de pobreza en que se emplazan las escuelas.

De esta manera, se han encontrado semejanzas y diferencias entre la valoración que los y las estudiantes han hecho de la enseñanza de ciertos saberes en sí y cómo piensan esos mismos saberes en relación al trabajo. La valoración sobre la preparación de la escuela en saberes desligados del trabajo, mostró que allí hay más encuentros que diferencias entre las miradas de estudiantes de escuelas ubicadas en zonas diferenciales. Los y las estudiantes expresaron cómo los saberes asociados a Buscar información y Valorar el esfuerzo por superarse son los que más circulan en las escuelas secundarias de Caleta Olivia, independientemente de las condiciones de pobreza en que esa escolarización sucede. Como se ha mencionado, el aprender a aprender y el saber ser se vuelven competencias que las escuelas son llamadas a enseñar, y esto es lo que efectivamente los y las jóvenes valoran. Ello nos habla de cómo los procesos de subjetivación en tiempos de gubernamentalidad neoliberal se hacen presentes en la formación de un Yo buscador y resiliente desde la escolarización secundaria (Grinberg, 2013).

Por otro lado, los circuitos escolares mostraron mayor diferenciación cuando los sentidos estudiantiles sobre los saberes escolares se colocan en relación al trabajo. Las consideraciones sobre el trabajo en grupo, el estudiar, el uso de tecnologías y la disciplina como saberes que las escuelas enseñan en relación al trabajo, es algo que tiene distinto peso según los niveles de pobreza en que se emplazan las escuelas. En relación a ello, el porcentaje de estudiantes que menciona que esas enseñanzas ocurren disminuye notablemente cuando se trata de jóvenes de escuelas ubicadas en los emplazamientos más pobres. Entre estos jóvenes prevaleció el No responde como expresión ante la pregunta por los saberes escolares que pueden ser útiles para el trabajo. Si bien entre la consideración de la enseñanza de saberes en sí y la enseñanza de saberes para el trabajo hubo una disminución en los y las estudiantes de todas las escuelas, en aquellos y aquellas que asisten a las escuelas de las zonas más pobres este hiato parece profundizarse aún más.

Estas incertidumbres no sorprenden en un contexto regional donde las dinámicas socio-laborales presentan las características inestables, fluctuantes, inciertas propias de una economía fuertemente condicionada por las lógicas flexibles del capital transnacional. Dicho de otra manera, las correspondencias con los circuitos escolares de la educación secundaria pública en Caleta Olivia nos muestran que éstos presentan al menos dos características. Por un lado, que la manera en que se configura la distribución diferencial y desigual de saberes para el trabajo ubica, claramente, a los y las estudiantes de las escuelas en NBI Alto como potenciales trabajadores de las zonas más periféricas del mercado de trabajo (Harvey, 2000), o directamente como los futuros supernumerarios (Castel, 1997). Por otro lado, aún para los y las estudiantes de las escuelas ubicadas en zonas menos pobres, las relaciones entre saberes escolares y trabajo se construyen bajo la sombra de la incertidumbre en cuanto a las utilidades laborales de los saberes que se están enseñando. Por eso tampoco sorprende que los saberes que los y las estudiantes expresaron que más y mejor se enseñan en las escuelas, aun cuando estos no aparezcan asociados al mundo laboral (o justamente por ello), sean aquellos que se dirigen a la formación de sujetos que se esfuercen en superarse a sí mismos y sujetos buscadores de información, de empleo, de oportunidades.

\section{Referencias bibliográficas}

Acconcia, M. y Álvarez, M. (2008). El ser ypefiano. Una identidad al abrigo de la Empresa (o una identidad territorializada). En: Ruiz, J.D. (Coord.), Petróleo y región austral: Saberes del trabajo y educación técnica. Reconfiguraciones y nuevas subjetividades (pp. 65-90). Buenos Aires, Argentina: Editorial Dunken.

Aranciaga, I. (2004). Tecnología y sociedad en el petróleo de la Patagonia argentina, (Tesis de Maestría). UNPA, Caleta Olivia, Argentina.

Armella, J. y Langer, E. (2020). De la ilusión al desencanto: sentidos y críticas en torno a la inclusión digital. Un estudio con docentes de escuelas secundarias emplazadas en contextos de pobreza urbana. Espacios en Blanco. Revista de Educación, 30, (1), 99-115

Ball, J., Maguire, M., Braum, A., Hoskins, K., y Perryman, J. (2012) How schools do Policy. Policy enactmentes in secondary schools. New York: Routledge.

Baudelot, C. (1998). El nivel educativo sube. Madrid: Morata.

Baudelot, C. y Establet, R. (1975). La escuela capitalista. Madrid: Siglo XXI Editores.

Benítez Larghi, S.; Lemus, M. y Welschinger Lascano, N. (2014). La inclusión masiva de tecnologías digitales en el ámbito escolar. Un estudio comparativo de la apropiación de TIC por estudiantes de clases populares y clases medias en el marco del Programa Conectar Igualdad en el Gran La Plata. Propuesta Educativa, 2, (42), 86 -92

Bowles, S. y Gintis, H. (1985). La instrucción escolar en la América capitalista. Madrid: Siglo XXI Editores

Braslavsky, C. (1985). La discriminación Educativa en Argentina. Grupo Editor Latinoamericano.

Braverman H. (1987). Trabajo y capital monopolista. México: Nuestro Tiempo.

Brunet, I. y Zavaro, B (2014). Competitividad, Competencias y fin del ciclo fordista. Revista Internacional de Sociología de la Educación, 3, (1),1-25

Carman, M.; Vieira da Cunha, N., \& Segura, R. (2013). Antropología, diferencia y segregación urbana. En Carman, M.; Vieira da Cunha, N. y Segura, R., Segregación y diferencia en la ciudad, 11-34. Quito: FLACSO-CLACSO

Castel, R. (1997). La metamorfosis de la cuestión social. Una crónica del salariado. Buenos Aires: Paidós.

Castro Gómez, S. (2010). Historia de la gubernamentalidad. Razón de Estado, liberalismo y neoliberalismo en Michel Foucault. Bogotá: Siglo del Hombre Editores.

Coriat, B. (2011). El taller y el robot. Ensayos sobre el fordismo y la producción en masa en la era de la electrónica. México D.F.: Siglo XXI.

De Ibarrola, M. (2016). Claroscuros en las relaciones entre la escolaridad y el trabajo. Configuraciones y límites. Revista Páginas de Educación, 9, (2). 
REVISTA DE LA ESCUELA DE CIENCIAS DE LA EDUCACIÓN, AÑO 17, NRO. 16, VOL. 2, JULIO A DICIEMBRE DE 2021. PÁGINAS 37-46. ISSN 2362-3349 (EN LÍNEA). LAS MIRADAS DE ESTUDIANTES DEL NIVEL SECUNDARIO SOBRE LOS SABERES PARA EL TRABAJO EN EL CAPITALISMO FLEXIBLE: UN ESTUDIO EN CALETA OLIVIA, SANTA CRUZ. MAURO VICTOR GUZMÁN.

De la Garza, E. (Coord). (2000). Tratado latinoamericano de sociologia del trabajo. México: El Colegio de México/ Facultad Latinoamericana de Ciencias Sociales/ Universidad Autónoma Metropolitana/ Fondo de Cultura Económica.

De Marinis, P. (2002). Ciudad, "cuestión criminal" y gobierno de poblaciones. Política y Sociedad, 39, (2), 319-338.

Feres, J. C. y Mancero, X. (2001). El método de las necesidades básicas insatisfechas (NBI) y sus aplicaciones en América Latina. Santiago de Chile: CEPAL

Figari, C. (2011). Procesos de formación, gestión por competencias y nuevas configuraciones profesionales. En Figari, C., Spinosa, M y Testa, J. (Comp) Trabajo y formación en debate. Saberes, itinerarios y trayectorias de profesionalización, 21-42. Buenos Aires: Fundación Centro de Integración, Comunicación, Cultura y Sociedad.

Foucault, M. (1989). Vigilar y castigar. Buenos Aires: Siglo XXI.

Foucault, M. (2007). Nacimiento de la biopolítica: curso en el Collège de France: 1978-1979. Buenos Aires: Fondo de Cultura Económica.

Grinberg, S. (2003). El mundo del trabajo en la escuela. La producción de Significados en los campos curriculares. Serie Cuadernos de Cátedra UNSAM. Buenos Aires: Jorge Baudino Ediciones.

Grinberg, S. (2008). Educación y poder en el Siglo XXI. Gubernamentalidad y Pedagogía en las sociedades de gerenciamiento. Buenos Aires: Miño y Dávila.

Grinberg, S. (2009). Pedagogía y gubernamentalidad en las sociedades del gerenciamiento. Notas de formación entre competencias y abyección. Espacios en Blanco. Revista de educación, 19,157-180.

Grinberg, S. (2013). Sociedad de la información, tecnologías y pedagogías de las competencias en la era del management. Hacia una genealogía. Revista Horizontes Sociológicos, 1, (2), 86-98.

Harvey, D. (2000). La condición de la posmodernidad. Investigación sobre los orígenes del cambio cultural. Buenos Aires: Amorrortu.

Harvey, D. (2020). Razones para ser anticapitalistas. Buenos Aires: CLACSO

Jacinto, C. (2013) La formación para el trabajo en la escuela secundaria como reflexión crítica y como recurso. Propuesta Educativa, 22, (40), 48-63.

Jacinto, C. y Millenaar, V. (2012). Los nuevos saberes para la inserción laboral. Formación para el trabajo con jóvenes vulnerables en Argentina. Revista Mexicana de Investigación Educativa, 17, (52),141-166.

Lazzarato, M. y Negri, A. (2001). Trabajo inmaterial. Formas de vida y producción de subjetividad. Rio de Janeiro: DPyA Editora.

Llomovatte, S. (1990). La educación media y el trabajo en la Argentina. Propuesta Educativa, № 3/4. Buenos Aires.

Márquez, D. (2017). Del petróleo estatal al petróleo privado: continuidades y rupturas en el mundo sociolaboral de los trabajadores petroleros de la Cuenca del Golfo San Jorge durante las últimas tres décadas. Ponencia presentada en XVI Jornadas Interescuelas Universidad de Mar del Plata.

Marx, K. (1990). El capital. Crítica de la economía política. México: Siglo XXI.

Miranda, A. y Arancibia, M. (2017). El futuro está incompleto: La construcción de trayectorias laborales sobre principios de siglo 21. Revista Trabajo y Sociedad, 28,195-217.

Noguera Ramírez, C. y Marín-Díaz, D. (2012). Educar es gobernar: La educación como arte de gobierno. Cuadernos de Pesquisa, (42), 14-29.

Olivera, R., Alfaro, A. y Estrada, S. (2012). Precariedad laboral y heterogeneidad ocupacional: una propuesta teóricometodológica. Revista Mexicana de Sociología, 74 (2), 213-243.

Pérez, A. (2013). Políticas de escolarización: Un acercamiento al análisis de la oferta escolar en clave de territorio. En Grinberg, S. (coord.) La escuela not dead. Dispositivos pedagógicos, territorios y desigualdad, 21-38. Rio Gallegos: Ediciones Universidad Nacional de la Patagonia Austral

Prado, M. y Robledo, M. (2010). La actividad hidrocarburífera en la Cuenca del Golfo San Jorge: El papel del empresariado regional. Revista de estudios regionales y mercado de trabajo, (6), 117-193.

Prevot-Schapira, M. F. (2001). Fragmentación espacial y social: conceptos y realidades. Revista Perfilaes Latinoamericanos, $019,33-56$

Rojo, S. y Rotondo, S. (2009). Perfil de especialización del empleo registrado en el Golfo San Jorge: un diagnóstico desde la perspectiva del desarrollo local. Actas de $9^{\circ}$ Congreso Nacional de Estudios del Trabajo, Buenos Aires.

Romero, J. y Galaretto, M. (2013). Nuevas tecnologías, nuevos escenarios educativos y laborales en la zona norte de Santa Cruz. Rio Gallegos: UNPA EDITA.

Ruiz, J. D., y Muñoz, N. (2008). La privatización de YPF y su repercusión en las subjetividades. En Ruiz, J.D. (Coord.), Petróleo y región austral: Saberes del trabajo y educación técnica. Reconfiguraciones y nuevas subjetividades, 91-118. Buenos Aires: Editorial Dunken.

Salvia, A. (1997). Crisis y reestructuración de complejos mineros: estudios de dos sistemas regionales patagónicos. En Salvia, A. y Panaia, M. (comp.), La Patagonia privatizada, 36-48. Buenos Aires: Colección CEA-CBC.

Saraví, G. (2015) Juventudes fragmentadas. Socialización, clase y cultura en la construcción de la desigualdad. México: FLACSO.

Schweitzer, A. (2012). Petróleo y territorios en la Provincia de Santa Cruz. Aproximaciones al estudio del espacio del Golfo de San Jorge. En Álvarez, M, Michniuk, N. y Villanueva, M. (coord.) Educación y trabajo, miradas desde lo regional. Territorio y desigualdad de oportunidades, 21-54. Buenos Aires: Editorial El Colectivo

Sennett, R. (2000). La corrosión del carácter. Las consecuencias personales del trabajo en el nuevo capitalismo. Barcelona: Anagrama.

Sennett, R. (2009). El artesano. Barcelona: Editorial Anagrama

Spinosa, M. (2006). Los saberes y el trabajo. Anales de la Educación Común, 2, (5), 164-173.

Tanguy L. (1986) L'introuvable relation formation-emploi, Paris, La Documentation française.

Vacca, C. y Shinelli,D. (2005). Caracterización económica de la provincia de Santa Cruz y la perspectiva de un nuevo perfil productivo. Actas de $7^{\circ}$ Congreso Nacional de Estudios del Trabajo, Buenos Aires.

Veiga, D (2009). Desigualdades sociales y fragmentación urbana. En Poggiese, H y Cohen Egler, T (comp.) Otro desarrollo urbano: ciudad incluyente, justicia social y gestión democrática, 51-61. Buenos Aires: CLACSO 